\title{
Equivelar octahedron of genus 3 in 3-space
}

\author{
Ruslan Mizhaev (mimodart@gmail.com) Apr. 2020
}

\begin{abstract}
Building up a toroidal polyhedron of genus 3, consisting of 8 nine-sided faces, is given. From the point of view of topology, a polyhedron can be considered as an embedding of a cubic graph with 24 vertices and 36 edges in a surface of genus 3 . This polyhedron is a contender for the maximal genus among octahedrons in 3-space.
\end{abstract}

\section{Introduction}

This solution can be attributed to the problem of determining the maximal genus of polyhedra with the number of faces $-f$. As is known, at least 7 faces are required for a polyhedron of genus $g=1$. For cases $f \geq$ 8 , there are currently few examples.

If all faces of the toroidal polyhedron are $p$ - gons and all vertices are q-valence $(q \geq 3)$, such polyhedral are called either locally regular or simply equivelar [1]. The characteristics of polyhedra are abbreviated as $\{p, q ; g\}[1]$.

\section{Polyhedron $\{9,3 ; 3\}$ V1.}

The paper considers building up a polyhedron $\{9,3 ; 3\}$ in 3 -space. The faces of a polyhedron are nonconvex flat 9-gons without self-intersections. The polyhedron is symmetric when rotated through $180^{\circ}$ around the $\boldsymbol{z}$ axis (Fig. 3).

One of the features of this polyhedron is that any face has two pairs with which it borders two edges. The polyhedron also has a ratio of angles and faces - $f=p-1$.

To describe polyhedra with similar characteristics $(f=p-1)$ we use the Euler formula $v-e+f=\chi=$ $2-2 g$, where $\chi$ is the Euler characteristic. Since $q=3$, the equality $3 v=2 e$ holds true. Therefore, the number of faces and genus:

$$
\begin{gathered}
g=\frac{f \cdot(f+1)}{12}-\frac{f}{2}+1 \\
f=\frac{\sqrt{48 g-23}+5}{2}
\end{gathered}
$$

Table 1 lists some of the first polyhedra that could possibly have similar characteristics for $f \geq 8$. At present, it is difficult to answer whether it is possible to realize $g=4$ and $g=8$.

$\underline{\text { Table } 1}$

\begin{tabular}{|c|c|c|c|c|}
\hline Genus, $\boldsymbol{g}$ & Faces, $\boldsymbol{f}$ & Vertices, $\boldsymbol{v}$ & Edges, $\boldsymbol{e}$ & Angles, $\boldsymbol{p}$ \\
\hline $\mathbf{3}$ & 8 & 24 & 36 & 9 \\
\hline $\mathbf{4}$ & 9 & 30 & 45 & 10 \\
\hline $\mathbf{8}$ & 12 & 52 & 78 & 13 \\
\hline
\end{tabular}



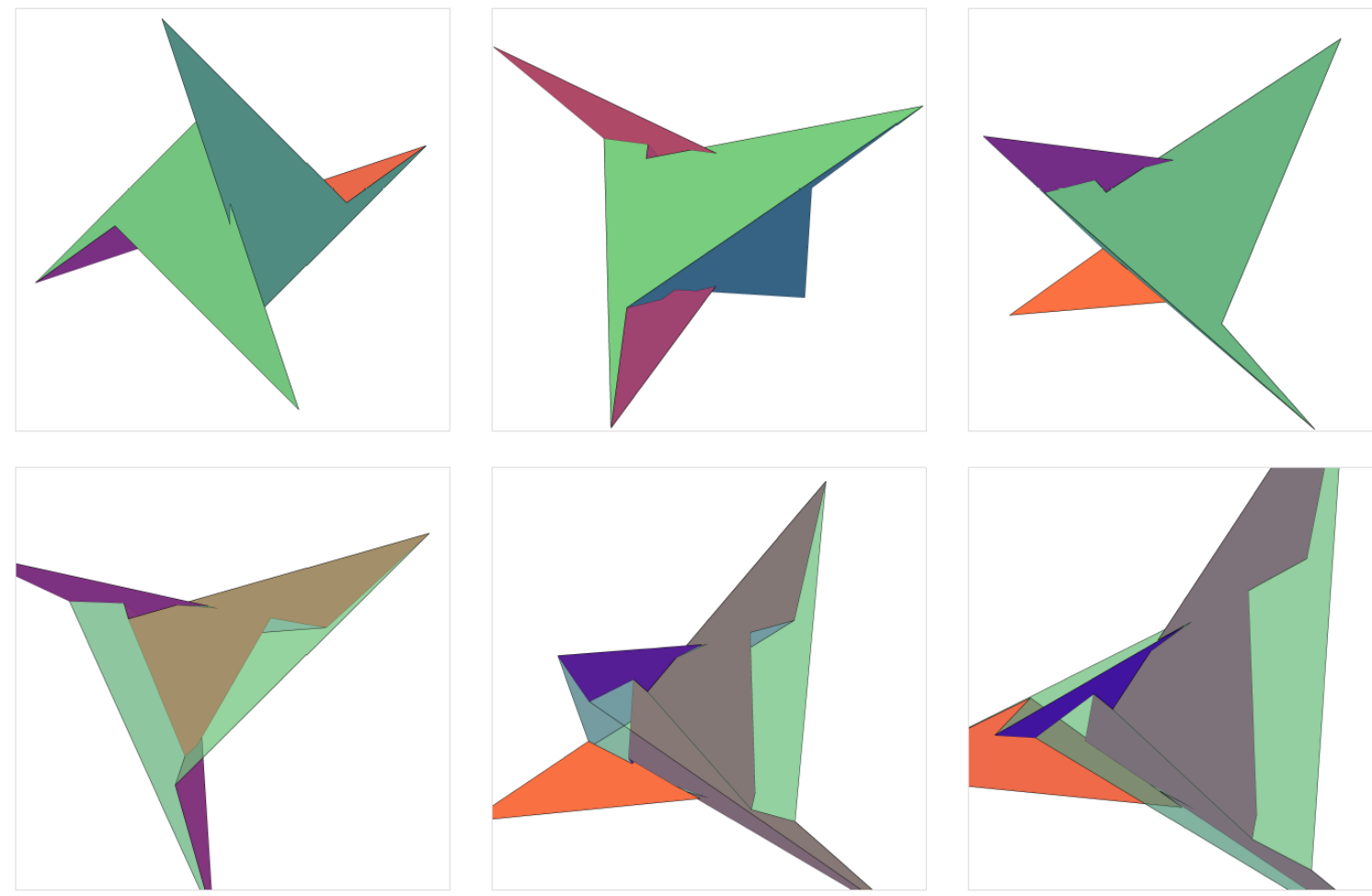

Fig. 1. General views $\{9,3 ; 3\}$ V1
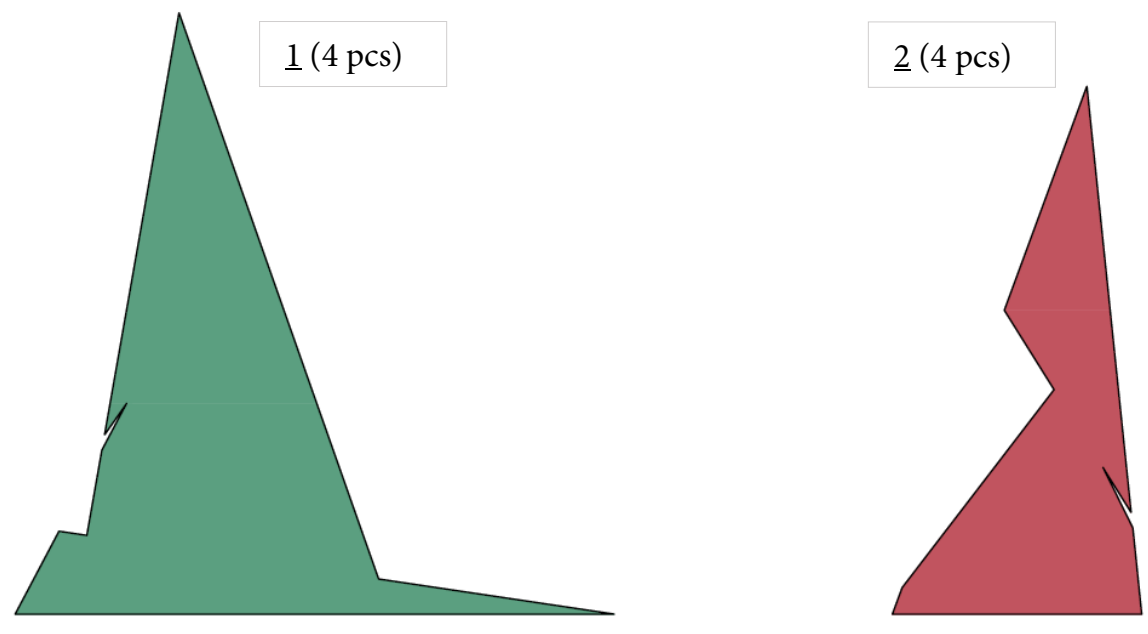

Fig. 2. Layout of faces on the plane $\{9,3 ; 3\}$ V1
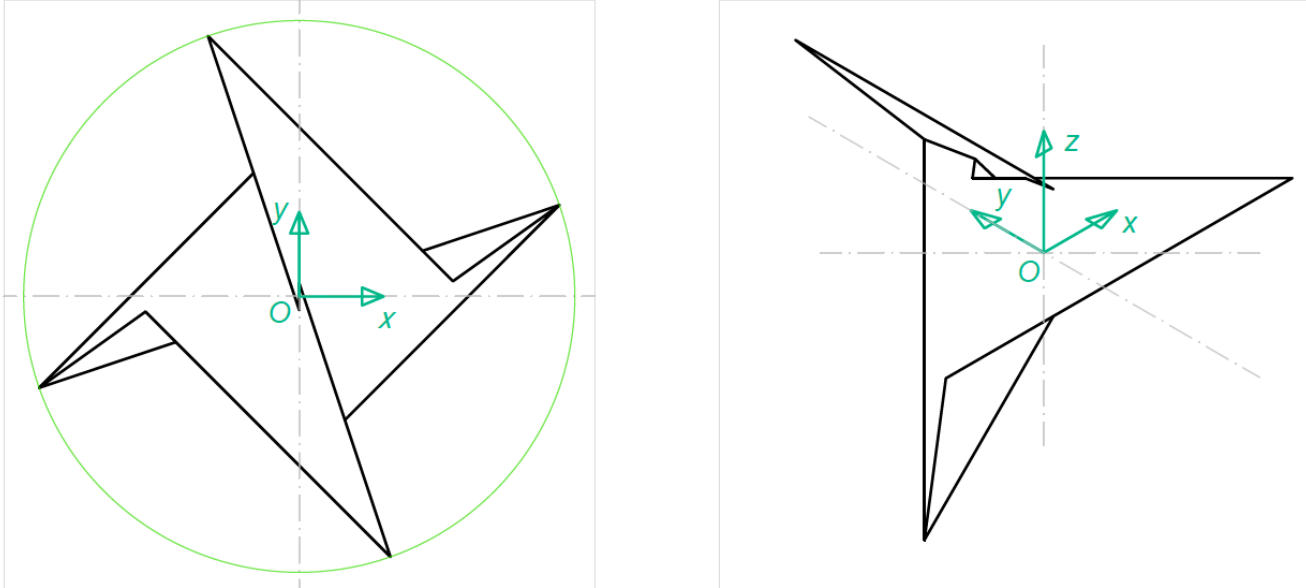

Fig. 3. Coordinate axis $\{9,3 ; 3\}$ V1 


\section{Vertex coordinates $\{9,3 ; 3\}$ V1}

Coordinates are represented in the Cartesian system. The polyhedron faces lie in the planes bounding the faces of 2 tetrahedrons (outer and inner faces). Below are the equations of the planes.

Planes 1 (Fig. 2). Tetrahedron 1

$y+z+100=0$

$x-z+100=0$

$y-z-100=0$

$x+z-100=0$
Planes $\underline{2}$ (Fig. 2). Tetrahedron 2

$x-7 y-4 z-384=0$
$7 x+y-4 z+384=0$
$x-7 y+4 z+384=0$
$7 x+y+4 z-384=0$
Coordinates of polyhedron vertices $\{9,3 ; 3\}$ V1

Table 2

\begin{tabular}{|c|c|c|c|}
\hline № & $\boldsymbol{x}$ - coordinates & $y$ - coordinates & $z$ - coordinates \\
\hline 1 & -308 & -108 & -208 \\
\hline 2 & 308 & 108 & -208 \\
\hline 3 & 16 & 0 & -100 \\
\hline 4 & -16 & 0 & -100 \\
\hline 5 & 28 & 4 & -96 \\
\hline 6 & -28 & -4 & -96 \\
\hline 7 & $-4272 / 41$ & $-448 / 41$ & $-3652 / 41$ \\
\hline 8 & 4272 / 41 & $448 / 41$ & $-3652 / 41$ \\
\hline 9 & -182 & -18 & -82 \\
\hline 10 & 182 & 18 & -82 \\
\hline 11 & $-1216 / 13$ & $-336 / 13$ & $-964 / 13$ \\
\hline 12 & $1216 / 13$ & $336 / 13$ & $-964 / 13$ \\
\hline 13 & $336 / 13$ & $-1216 / 13$ & $964 / 13$ \\
\hline 14 & $-336 / 13$ & $1216 / 13$ & $964 / 13$ \\
\hline 15 & 18 & -182 & 82 \\
\hline 16 & -18 & 182 & 82 \\
\hline 17 & $448 / 41$ & $-4272 / 41$ & 3652 / 41 \\
\hline 18 & $-448 / 41$ & $4272 / 41$ & 3652 / 41 \\
\hline 19 & -4 & 28 & 96 \\
\hline 20 & 4 & -28 & 96 \\
\hline 21 & 0 & -16 & 100 \\
\hline 22 & 0 & 16 & 100 \\
\hline 23 & -108 & 308 & 208 \\
\hline 24 & 108 & -308 & 208 \\
\hline
\end{tabular}

\section{Coordinates of face vertexes $\{9,3 ; 3\}$ V1}

Table 3

\begin{tabular}{|c|c|c|c|c|c|c|c|c|c|c|}
\hline \multirow{2}{*}{ Face number } & \multicolumn{9}{|c|}{ Vertex number in table 2 } \\
\cline { 2 - 13 } & $\mathbf{1}$ & $\mathbf{2}$ & $\mathbf{3}$ & $\mathbf{4}$ & $\mathbf{5}$ & $\mathbf{6}$ & $\mathbf{7}$ & $\mathbf{8}$ & $\mathbf{9}$ \\
\hline $\mathbf{1}$ & 1 & 3 & 5 & 6 & 7 & 9 & 12 & 14 & 18 \\
\hline $\mathbf{2}$ & 2 & 10 & 13 & 15 & 17 & 20 & 21 & 22 & 23 \\
\hline $\mathbf{3}$ & 8 & 12 & 13 & 16 & 18 & 19 & 20 & 21 & 23 \\
\hline $\mathbf{4}$ & 2 & 4 & 5 & 6 & 8 & 10 & 11 & 13 & 17 \\
\hline $\mathbf{5}$ & 1 & 4 & 3 & 5 & 8 & 10 & 12 & 16 & 23 \\
\hline $\mathbf{6}$ & 1 & 9 & 14 & 16 & 18 & 19 & 22 & 21 & 24 \\
\hline $\mathbf{7}$ & 7 & 11 & 14 & 15 & 17 & 19 & 20 & 22 & 24 \\
\hline $\mathbf{8}$ & 2 & 3 & 4 & 6 & 7 & 9 & 11 & 15 & 24 \\
\hline
\end{tabular}




\section{Polyhedron graph $\{9,3 ; 3\}$ V1}

A polyhedron can be described as an embedding of a cubic graph in a surface of genus 3 in 3-space. More detailed characteristics of the graph are given in table 4 .

\section{Table 4}

\begin{tabular}{|l|c|}
\hline Vertices & 24 \\
\hline Edges & 36 \\
\hline Radius & 5 \\
\hline Diameter & 5 \\
\hline Chromatic number & 3 \\
\hline Chromatic index & 3 \\
\hline Girth & 4 \\
\hline Automorphisms [8] & 8 \\
\hline Hamiltonian cycle & yes \\
\hline
\end{tabular}

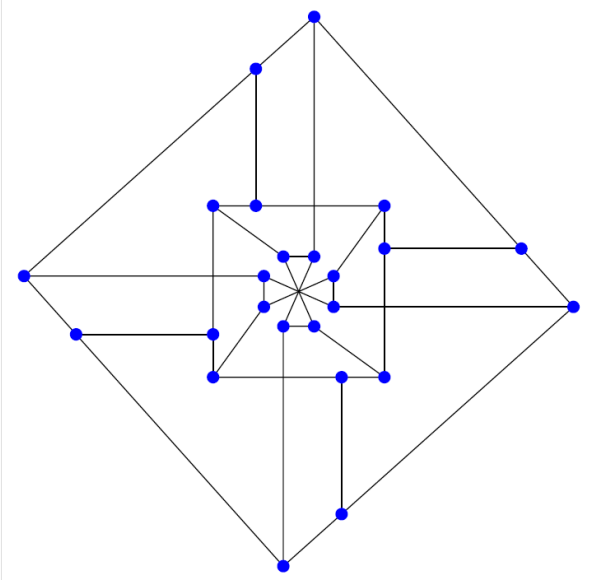

Fig. 4. Basic view of the graph $\{9,3 ; 3\}$ V1

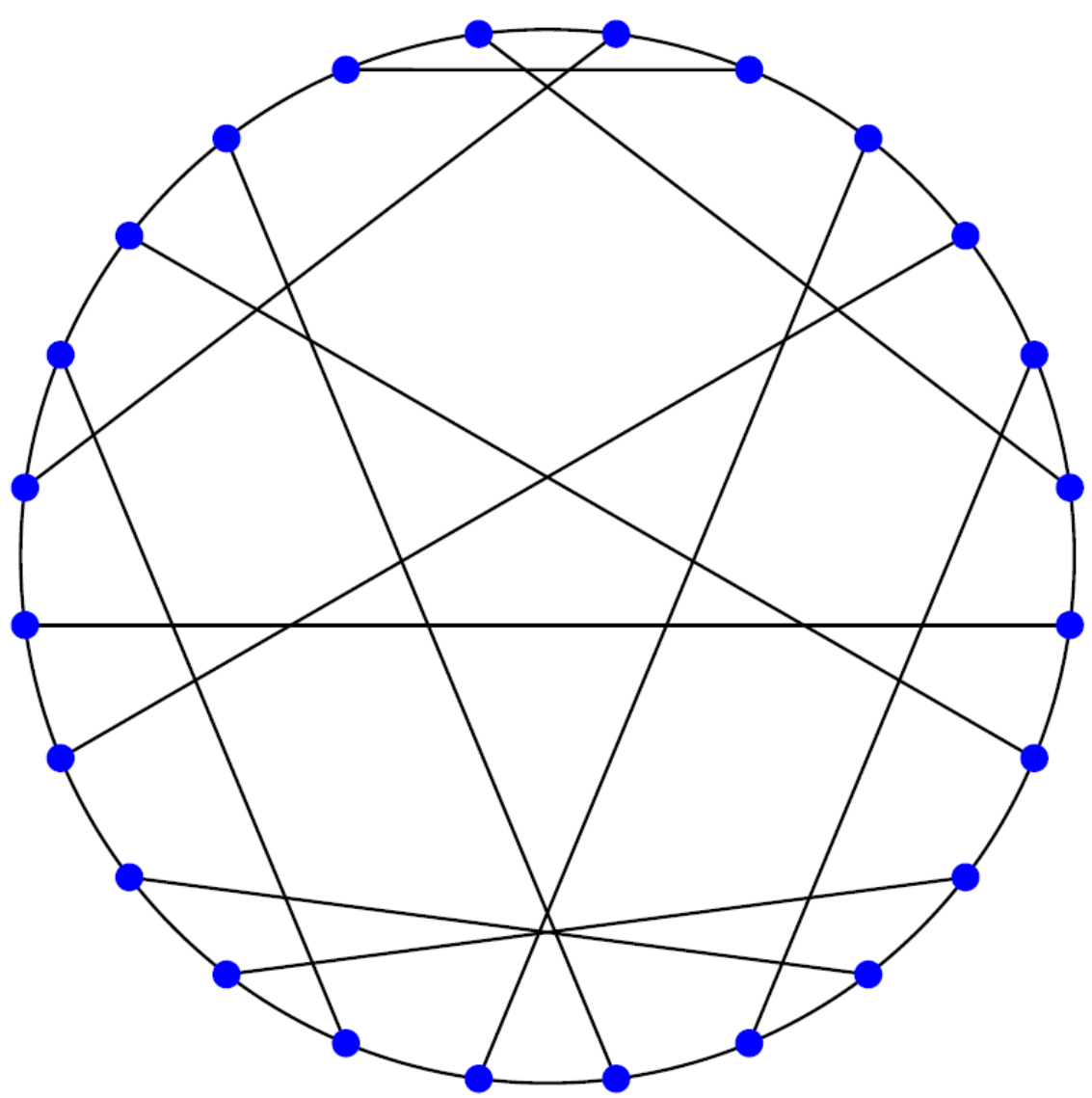

Fig. 5. View with Hamiltonian cycle $\{9,3 ; 3\}$ V1 


\section{Polyhedron $\{9,3 ; 3\}$ V2.}

The combinatorial structure of this polyhedron differs from $\{9,3 ; 3\}$ V1. Each face has one pair with which it borders two edges, also any two faces have a common edge like the tetrahedron and Szilassi polyhedron.
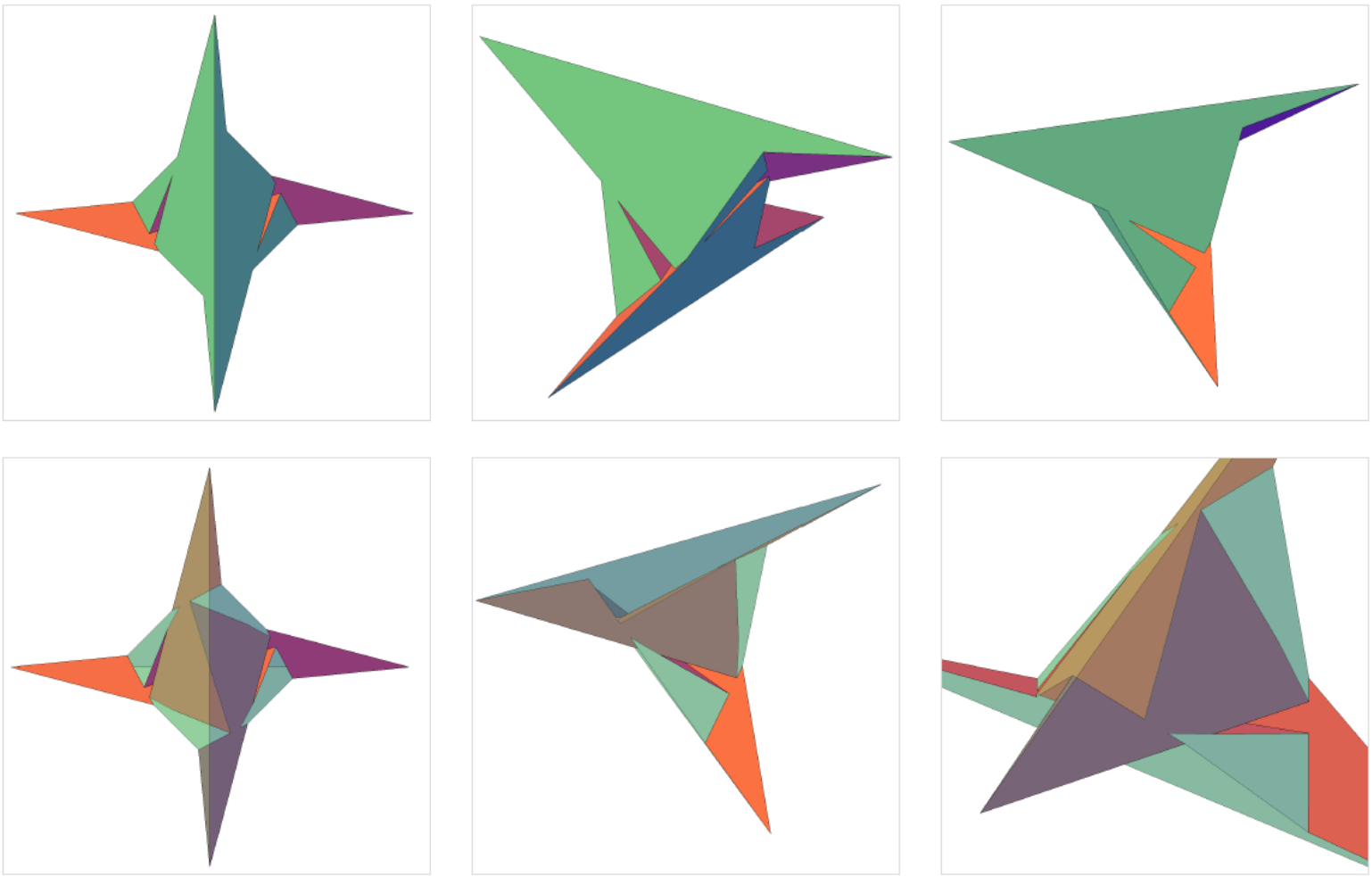

Fig. 6. General views $\{9,3 ; 3\}$ V2
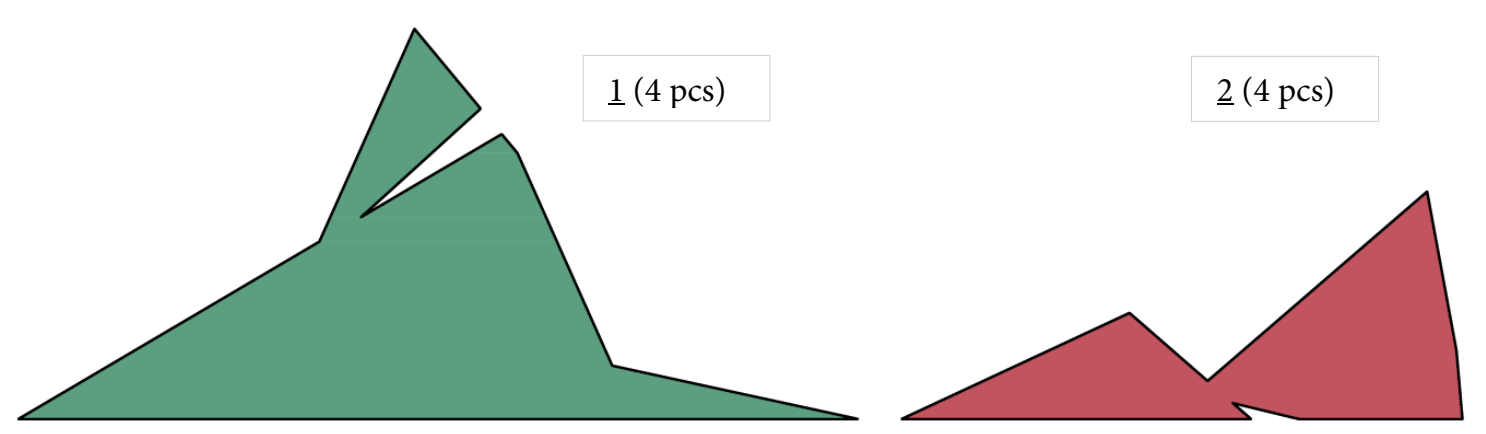

Fig. 7. Layout of faces on the plane $\{9,3 ; 3\}$ V2
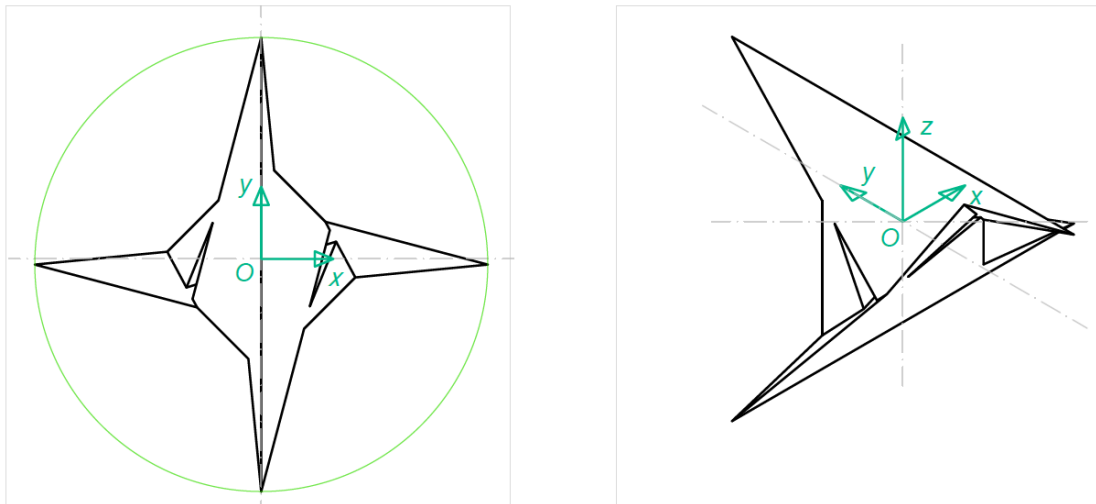

Fig. 8. Coordinate axis $\{9,3 ; 3\}$ V2 
Planes $\underline{1}$ (Fig. 7). Tetrahedron 1

$2 y+z+100=0$

$2 x-z+100=0$

$2 y-z-100=0$

$2 x+z-100=0$
Planes $\underline{2}$ (Fig. 7). Tetrahedron 2

$15 x-49 y-53 z-2120=0$

$49 x+15 y-53 z+2120=0$

$15 x-49 y+53 z+2120=0$

$49 x+15 y+53 z-2120=0$

Coordinates of polyhedron vertices $\{9,3 ; 3\}$ V2

$\underline{\text { Table } 5}$

\begin{tabular}{|c|c|c|c|}
\hline № & $x$ - coordinates & $y$ - coordinates & $z$ - coordinates \\
\hline 1 & -212 & 0 & -100 \\
\hline 2 & 212 & 0 & -100 \\
\hline 3 & -88 & 12 & -76 \\
\hline 4 & 88 & -12 & -76 \\
\hline 5 & -70 & $-150 / 7$ & -40 \\
\hline 6 & 70 & $150 / 7$ & -40 \\
\hline 7 & $-2120 / 33$ & $-1060 / 33$ & $-940 / 33$ \\
\hline 8 & $2120 / 33$ & $1060 / 33$ & $-940 / 33$ \\
\hline 9 & -60 & -40 & -20 \\
\hline 10 & 60 & 40 & -20 \\
\hline 11 & $-6360 / 163$ & $-7420 / 163$ & $-1460 / 163$ \\
\hline 12 & $6360 / 163$ & $7420 / 163$ & $-1460 / 163$ \\
\hline 13 & $-7420 / 163$ & $6360 / 163$ & $1460 / 163$ \\
\hline 14 & $7420 / 163$ & $-6360 / 163$ & $1460 / 163$ \\
\hline 15 & -40 & 60 & 20 \\
\hline 16 & 40 & -60 & 20 \\
\hline 17 & $1060 / 33$ & $-2120 / 33$ & $940 / 33$ \\
\hline 18 & $-1060 / 33$ & $2120 / 33$ & $940 / 33$ \\
\hline 19 & $-150 / 7$ & 70 & 40 \\
\hline 20 & $150 / 7$ & -70 & 40 \\
\hline 21 & 12 & 88 & 76 \\
\hline 22 & -12 & -88 & 76 \\
\hline 23 & 0 & 212 & 100 \\
\hline 24 & 0 & -212 & 100 \\
\hline
\end{tabular}

Coordinates of face vertexes $\{9,3 ; 3\}$ V2

Table 6

\begin{tabular}{|c|c|c|c|c|c|c|c|c|c|}
\hline \multirow{2}{*}{ Face number } & \multicolumn{9}{|c|}{ Vertex number in table 5} \\
\hline & 1 & 2 & 3 & 4 & 5 & 6 & 7 & 8 & 9 \\
\hline 1 & 1 & 3 & 5 & 6 & 7 & 9 & 11 & 14 & 17 \\
\hline 2 & 7 & 11 & 13 & 15 & 18 & 19 & 20 & 21 & 23 \\
\hline 3 & 4 & 6 & 8 & 10 & 14 & 16 & 21 & 23 & 24 \\
\hline 4 & 1 & 2 & 4 & 9 & 11 & 16 & 17 & 20 & 22 \\
\hline 5 & 8 & 12 & 14 & 16 & 17 & 19 & 20 & 22 & 24 \\
\hline 6 & 2 & 1 & 3 & 10 & 12 & 15 & 18 & 19 & 21 \\
\hline 7 & 3 & 5 & 7 & 9 & 13 & 15 & 22 & 24 & 23 \\
\hline 8 & 2 & 4 & 5 & 6 & 8 & 10 & 12 & 13 & 18 \\
\hline
\end{tabular}


Polyhedron graph $\{9,3 ; 3\}$ V2

The characteristics of the graph are shown in table 7.

$\underline{\text { Table } 7}$

\begin{tabular}{|l|c|}
\hline Vertices & 24 \\
\hline Edges & 36 \\
\hline Radius & 4 \\
\hline Diameter & 4 \\
\hline Chromatic number & 3 \\
\hline Chromatic index & 3 \\
\hline Girth & 5 \\
\hline Automorphisms [8] & 4 \\
\hline Hamiltonian cycle & yes \\
\hline
\end{tabular}

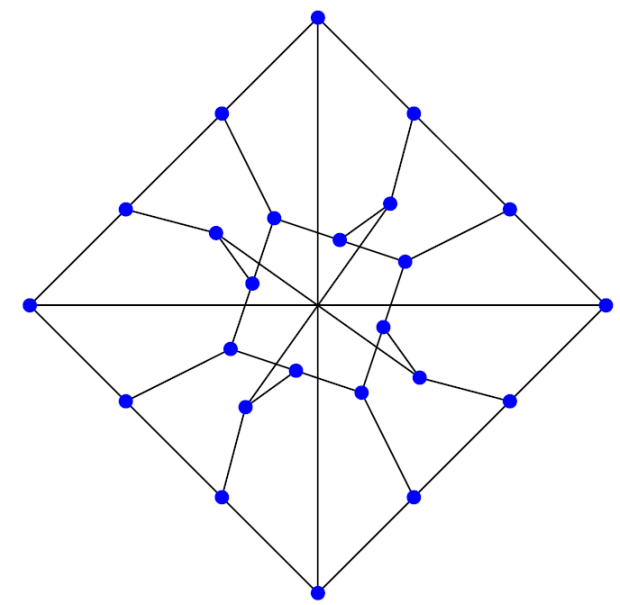

Fig. 9. Basic view of the graph $\{9,3 ; 3\}$ V2

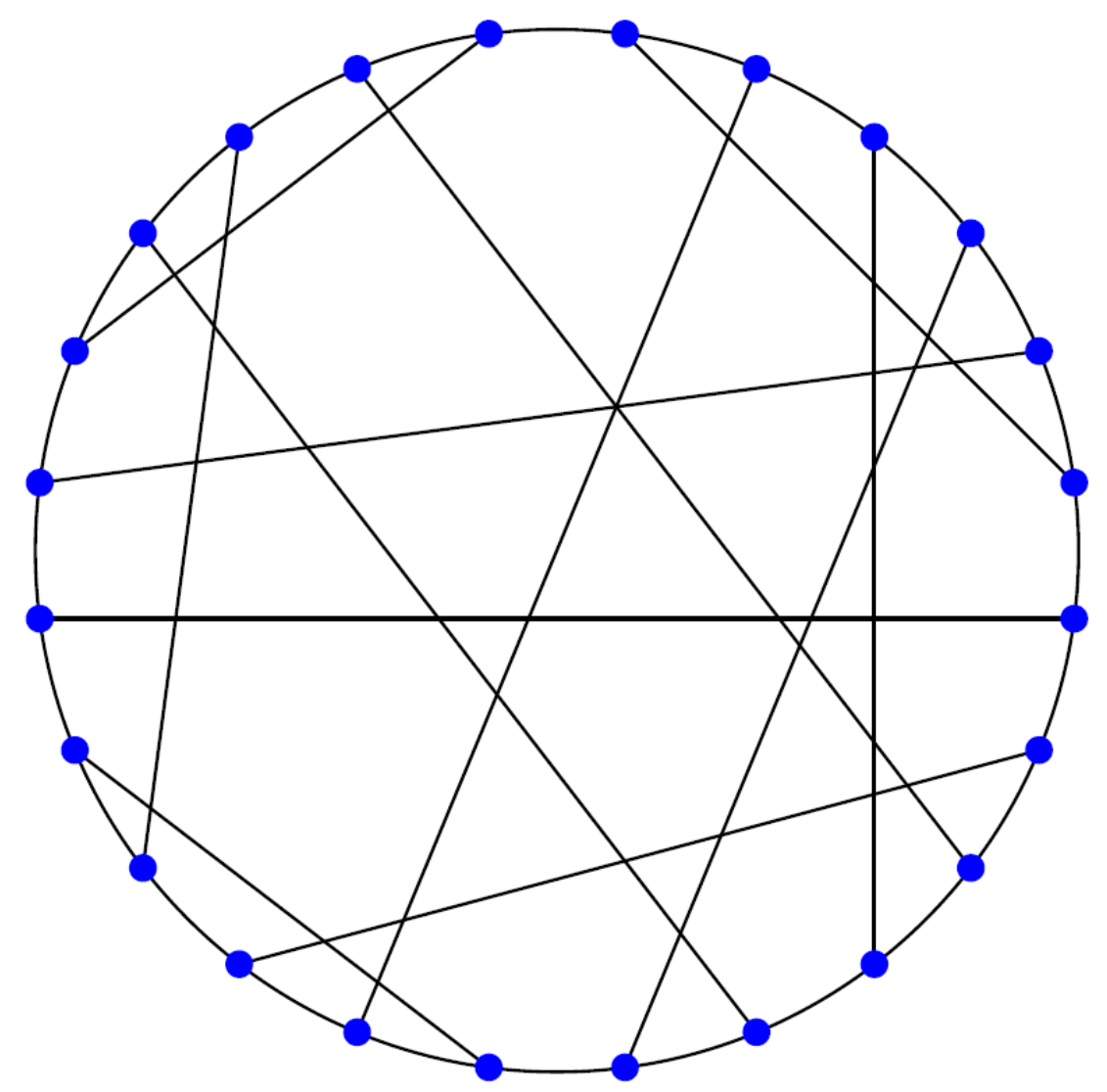

Fig. 10. View with Hamiltonian cycle $\{9,3 ; 3\}$ V2 


\section{Notes}

More images of the polyhedron are available at the link [11]

\section{References}

[1] Wills, Jörg. (2008). A locally regular heptagon-dodecahedron embedded in 3-space. European Journal of Combinatorics. 29. 1952-1955. 10.1016/j.ejc.2008.01.014

[2] G'abor G'evay, J"org M. Wills. On regular and equivelar Leonardo polyhedra. Ars Mathematica Contemporanea $\cdot$ January 2013. DOI: 10.26493/1855-3974.219.440

[3] Bokowski, Jürgen \& Pilaud, Vincent. (2014). Enumerating topological (n_k)-configurations. Computational Geometry. 47. 175-186. 10.1016/j.comgeo.2012.10.002.

[4] J.M. Wills. On Polyhedra with Transitivity Properties. Discrete \& Computational Geometry volume 1, pages195-199(1986).

[5] Gévay, Gábor \& Schulte, Egon \& Wills, Joerg. (2014). The Regular Grünbaum Polyhedron of Genus 5. Advances in Geometry. 14. 465-482. 10.1515/advgeom-2013-0033.

[6] Bokowski, Jürgen. (1989). A Geometric Realization Without Self-Intersections Does Exist for Dyck's Regular Map. Discrete \& Computational Geometry. 4. 583-589. 10.1007/BF02187748.

[7] Branko Grünbaum, Lajos Szilassi. Geometric realizations of special toroidal complexes/ Contributions to Discrete Mathematics, Vol 4 No 1 (2009), pages 21-39. 2009. ISSN 1715-0868.

[8] McKay, B.D. and Piperno, A., Practical Graph Isomorphism, II, Journal of Symbolic Computation, 60 (2014), pp. 94-112, http://dx.doi.org/10.1016/j.jsc.2013.09.003

[9] Serge Lawrencenko. Geometric realization of toroidal quadrangulations without hidden symmetries. arXiv:1307.1054 [math.MG]

[10] Szilassi, Lajos (1986), "Regular toroids", Structural Topology, 13: 69-80

[11] Mizhaev, Ruslan. 2020. "Toroidal polyhedra of a large genus." OSF. April 25. doi:10.17605/OSF.IO/F3G85. 